\title{
Performance Evaluation of IEEE 802.11e EDCA using QUALNET
}

\author{
P. Vijaya Kumari \\ PG Scholar, Department of CSE, \\ JNTUA College of Engineering (Autonomous), \\ Pulivendula, A.P., India.
}

\author{
P. Chenna Reddy \\ Professor, Department of CSE, \\ JNT University, \\ Anantapuramu, A.P., India.
}

\begin{abstract}
Wireless LANs are one of the fastest growing wireless technologies. IEEE 802.11 is a standard for Wireless LANs. This doesn't provide Quality of Service (QoS) for multimedia applications. IEEE 802.11e enhances the QoS in Wireless LANs. It introduces the Access-Categories (AC) by using Enhanced Distributed Channel Access (EDCA). It is a MAC protocol supporting service differentiation through different ACs. EDCA offers different priorities for different traffics. Every station contains four transmission queues one for each AC. Every AC has its own channel access parameters such as AIFS (Arbitration interface spacing), CW (Contention window min and max) and TXOP (Transmission opportunity). In this paper performance of IEEE 802.11e EDCA is evaluated in Qualnet 7.3 through simulations
\end{abstract}

\section{Keywords}

802.11e, QoS, Access categories, CW, TXOP, EDCA, AIFS

\section{INTRODUCTION}

\subsection{Overview of DCF and PCF}

The basic MAC protocol in IEEE 802.11 Distributed Coordination Function (DCF) [1] works under CSMA/CA (carrier sense multiple access with collision avoidance) protocol. DCF offers same preference to all devices to access channel in a given radio range [2]. A station first sense the channel before transmitting. The channel is sensed for Distributed Inter Frame Spacing (DIFS) and if it is idle then station can send the frames. The Station activates random backoff-counter before transmitting. The backoff-counter is decreased until channel is idle and stopped when a station detected transmission. If the station finds the idle channel again then the backoff-counter is reactivated. DCF backoffcounter is reduced by 1 from first slot of interval of DIFS [3]. A Station begins the sending of frames whenever the backoffcounter reaches zero. The backoff-counter time is selected from the range $(0, \mathrm{CW}-1)$. For every failure transmission $\mathrm{CW}$ is changed as $\mathrm{CW}_{\text {new }}=\left(\mathrm{CW}_{\text {old }}+1\right)^{*} \mathrm{PF}-1$ (where $\left.\mathrm{PF}=2\right)$ until CWmax (where CWmax =1023) [4]. If two stations backoffcounter reaches zero then collisions will occur.

Two access techniques are used to avoid collisions. Basic access method is, when the sender gains the channel access, it will send data directly and wait for acknowledgement (ACK) from the receiver. Whenever collision occurs, it will waste the entire time until the large packet transmission finishes. Another is Request-To-Send and Clear-To-Send (RTS/CTS) access method. A station gains the opportunity to access the channel, it first sends RTS and waits for CTS, and then starts transmission of packets. Other stations listening to RTS, CTS or data frames will defer a period of time to access the medium, called NAV (Network Allocation Vector). The NAV value is set according to the length field in the RTS, CTS frames.

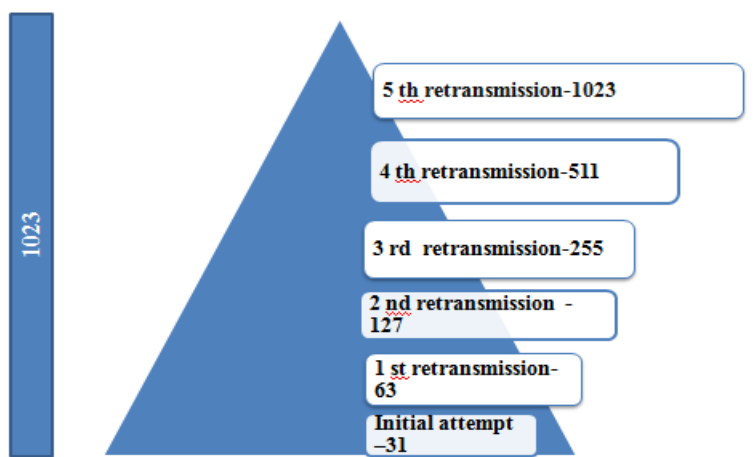

Fig 1: Exponential Increase of $\mathbf{C W}$

Point Coordination Function (PCF) - Point Coordinator (PC) is used in PCF to control channel access, and can support limited QoS. PC is always an Access Point (AP). PCF, a polling based mechanism, provides contention-free transmission in infrastructure network by using PC, usually in the AP to determine which station gains the channel access. DCF is transmitted during the Contention-Period (CP) and PCF is transmitted during the Contention Free-Period (CFP).

\subsection{Overview of EDCA}

To support QoS in Wireless LANs, the IEEE 802.11 Working Group proposed IEEE 802.11e [5]. The standard includes HCF (Hybrid Coordination Function). It contains two MAC mechanisms called EDCA and HCCA (Hybrid Coordination Channel Access). EDCA allows traffic differentiation for the stations in the network and differentiates four ACs by varying 4 EDCA parameters such as AIFS, minimal and maximal CW (CWmin and CWmax), and TXOP. Every station contains 4 transmission queues named AC_VO, AC_VI, AC_BE, and AC_BK and, for Voice traffic, video, Best-Effort, and Background respectively, where AC_BK has the lowerpriority and AC_VO has the higher-priority [6]. Every AC has its own parameter sets and backoff-counters as shown in Fig 2. The priority-level of an AC is decided based on AIFS and $\mathrm{CW}$ values shown in Table 1 . AC contains smaller AIFS, smaller CW values and larger TXOP for an AC. This represents that, it has a higher-priority $\mathrm{AC}$ than the other ACs.The IEEE 802.11e framework adds a mechanism for TXOP and the units are seconds. In TXOP, when packet transmission fails, the packets must be retransmitted to the channels [7]. 
Table.1: EDCA parameters

\begin{tabular}{|c|c|c|l|c|c|}
\hline & Priority & $\mathbf{C w}_{\text {min }}$ & $\mathbf{C w}_{\text {max }}$ & AIFS & TXOP \\
\hline AC-VO & AC[3] & 7 & 15 & 2 & $0.00308 S$ \\
\hline AC-VI & AC[2] & 15 & 31 & 2 & $0.006016 S$ \\
\hline AC_BE & AC[1] & 31 & 1023 & 3 & 0 \\
\hline AC_BK & AC[0] & 31 & 1023 & 7 & 0 \\
\hline
\end{tabular}

AIFS: AIFS is the minimal waiting time of a station, when it detect the channel idle for this time it can start its backoffcounter.

$\mathbf{C W}_{\min }$ and $\mathbf{C W}_{\text {max }}$ : The backoff-counter is selected from $\mathrm{CW}$. The backoff-counter is a random integer select from a interval $[0, \mathrm{CW}] . \mathrm{CW}_{\min }$ and $\mathrm{CW}_{\max }$ are the lower and upper boundaries of $\mathrm{CW} ; \mathrm{CW}_{\min }$ and $\mathrm{CW}_{\max }$ initialize first according to the $\mathrm{AC}$, but after each failure transmission, the backoffcounter window size is changed with use of $\mathrm{CW}_{\min }$.

Table 2: CW boundaries

\begin{tabular}{|l|l|l|}
\hline AC & MIN & MAX \\
\hline AC_VO & $($ CWmin+1)/4-1 & $($ CWmin+1)/2-1 \\
\hline AC_VI & $($ CWmin+1)/2-1 & CWmin \\
\hline AC_BE & CWmin & CWmax \\
\hline AC_BK & CWmin & CWmax \\
\hline
\end{tabular}

TXOP: TXOP is defined as beginning time and maximum duration, when a station starts to initiate the transmission.

An AC finds the channel idle for a period called AIFS declared by the corresponding $\mathrm{AC}$, then it decrements it's backoff-counter. After minimum waiting of AC for AIFS, every $\mathrm{AC}$, set its backoff-counter to a random number choose from the interval $[1, \mathrm{CW}+1]$. Backoff-counter is reduced by 1 from last slot of interval of AIFS [9].

Fig 2 indicates IEEE 802.11e with four Backoff-counters.

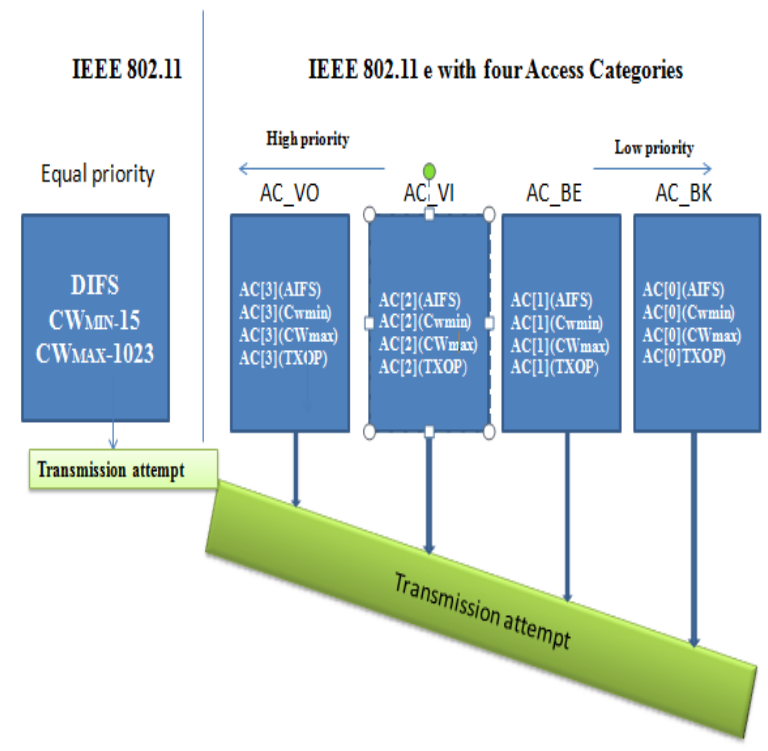

Fig 2: IEEE 802.11e with four Backoff-counters.

\section{LITERATURE SURVEY}

S. Kupa , R.Prakash [2], evaluated the throughput and delay of DCF with different packet HCCA rates. They assume fixed collision probability for each station, and evaluate the performance of the DCF. They proved how DCF will give equal preference to all traffics.

In [5], authors described a markov chain model to analyze performance of EDCA via simulations. This paper discussed how Call admission control (CAC) model is applied to get better QoS. In [7], authors show the performance evaluation for EDCA via simulations. They compare DCF and EDCA. In [9], a new dynamic scheme is implemented for the adaptation of the $\mathrm{CW}_{\min }$ to improve the service differentiation for 802.11e Wireless-LANs. They enhance the EDCA scheme by an algorithm which enables each station to modify the size of the $\mathrm{CW}_{\min }$ at run time which is used in its backoff algorithm at run time.

In [11], authors used the distributed advanced control algorithm to provide QoS. Resource reservation algorithm is used to send the traffic. They compare the modified results with original 802.11e's access methods and proved modified results are better than the original. Saeed Rashwand, and Vojislaav Mi_sic [12], proved that the impact of QoS differentiation parameters such as TXOP, AIFS and CWmin on EDCA performance. They used the non-zero TXOP and increasing the TXOP to all AC's will get some benefit to the lower-priority AC's.

Abdallah Shami, Jelena Mi_si [13], presented the Enhanced collision avoidance (ECA) scheme to improve voice transmissions when EDCA is used. Simulations show improvement in voice performance without affecting other traffic categories. In [14], authors described how various values of EDCA provide differentiation to gain channel access for different traffics through simulations and comparison of EDCA and DCF. Smaller CW size will lead to higher collisions and droppings of packets. The authors tune CW to get the better results.

\section{SIMULATIONS}

\subsection{Simulation Environment}

Qualnet 7.3 is used to simulate the EDCA. The scenario contains 50 nodes and all nodes are mobile nodes. Here 10 nodes are transmitting nodes. The parameters used for the simulations are given in table 4 . Table 3 show different types of bandwidths for different traffics.

Table 3: Minimum Bandwidths of ACs

\begin{tabular}{|l|l|}
\hline Instant message & $1,000 \mathrm{bps}$ \\
\hline voice over IP (VoIP) & $56 \mathrm{Kbps}$ \\
\hline video (480p) & $1 \mathrm{Mbps}$ \\
\hline HD video (720p) & $4 \mathrm{Mbps}$ \\
\hline HDX (1080p) & $7 \mathrm{Mbps}$ \\
\hline
\end{tabular}

Fig. 3 shows the scenario that is used in simulations. The scenario contains the 50 nodes connecting with wireless network. 10 nodes are transmitting nodes and remaining modes are receivers. Each transmitting nodes contains 4 types of traffics such as Voice traffic, Video, Best-Effort and Background 


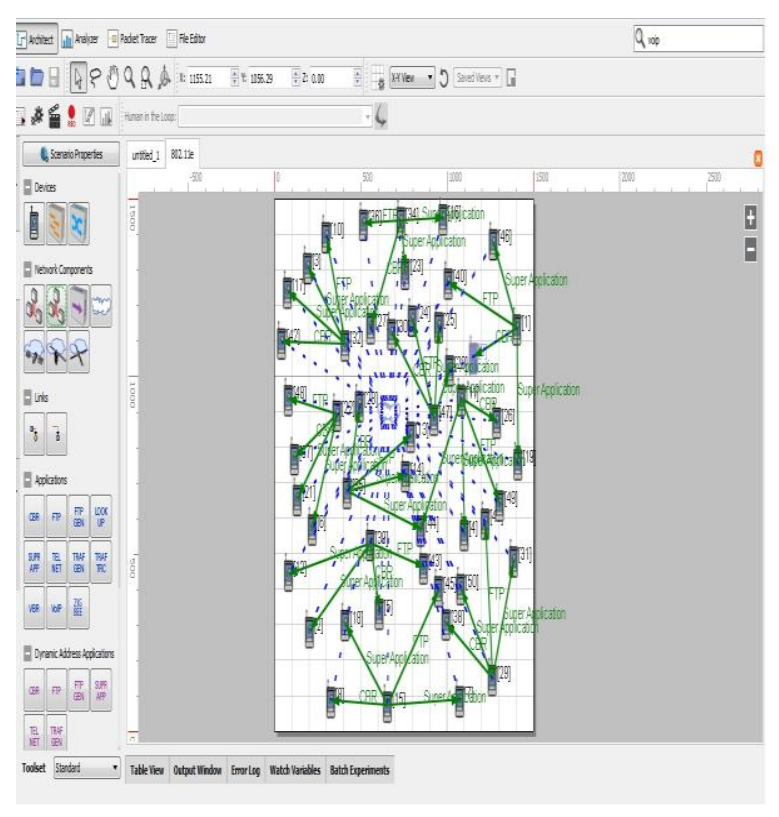

Fig. 3: Simulation scenario

Table 4 shows the simulation parameters and table 5 shows the data rates of four ACs used in simulation.

Table 4: Simulation parameters

\begin{tabular}{|c|c|}
\hline Radio-Type & $802.11 \mathrm{~b}$ \\
\hline Data rate & $2 \mathrm{Mbps}$ \\
\hline Mac protocol & $802.11 \mathrm{e}$ \\
\hline Routing protocol & Bellman Ford \\
\hline Mobility & Random way-point \\
\hline Traffic types & Super application, CBR, FTP \\
\hline Packet size & 512 bytes \\
\hline Number of nodes & 50 \\
\hline AIFS & $2,2,3,7$ \\
\hline CW & $7,15,31,31$ \\
\hline CW $_{\text {MAX }}$ & $15,31,1023,1023$ \\
\hline AIFS & $0.003008,0.006016,0,0$ \\
\hline
\end{tabular}

Table 5: Data Rates of AC s

\begin{tabular}{|c|c|c|}
\hline AC & Traffic & Data rate \\
\hline AC_VO & Super application(G.711) & 64 Kbps \\
\hline AC_VI & Super application((H.26) & 1 Mbps \\
\hline AC_BE & CBR & 512 bytes \\
\hline AC_BK & FTP & 512 bytes \\
\hline
\end{tabular}

\section{Super Application Traffic Generator:}

Super Application is a generic traffic generator. Super Application can simulate both UDP and TCP flows. Two-way flow (request-response) is supported for UDP based applications. Request packets move from source to destination, response packets travel from destination to source.

Command Line Configuration:

To use Super Application, the following format is needed:

SUPER-APPLICATION $<$ src $><$ dest $><$ keyword $1><$ value $1>$

\subsection{Performance Metrics}

1. End-to-End (E2E) Delay is the travelling time of a packet to travel from source to destination.

2. Throughput is the rate at which a network sends or receives data. Units are bits/sec.

3. Jitter: It is the differentiation between maximum and minimum delay of packets.

\subsection{Simulation Results and Analysis}

It can be observed from Fig. 4 that higher-priority AC gets higher throughput and lower-priority AC gets low throughput. When simulation-time increases $\mathrm{AC}[3]$ and $\mathrm{AC}[2]$ i.e. Voice, video throughput is increasing, remaining $\mathrm{AC}$ 's i.e. BestEffort, Background throughputs are decreased.

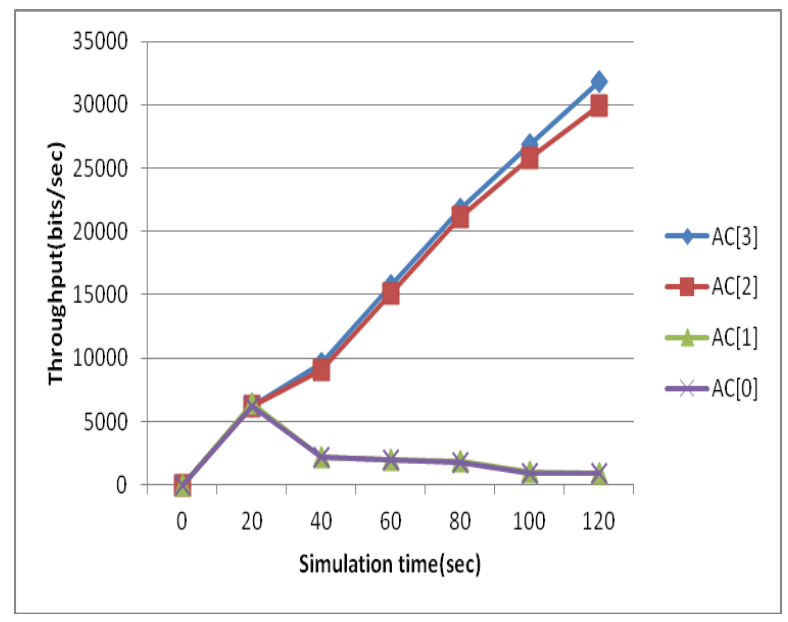

Fig 4: Simulation time Vs Throughput

It is shown in Fig 5 and 6 that higher-priority AC's get low jitter and E2E Delay, lower-priority AC gets high jitter and high E2E delay. When simulation-time increases $\mathrm{AC}[1]$, $\mathrm{AC}[0], \mathrm{E} 2 \mathrm{E}$ delay and jitter are increasing, no change in $\mathrm{E} 2 \mathrm{E}$ delay and jitter of $\mathrm{AC}[3], \mathrm{AC}[2]$. 


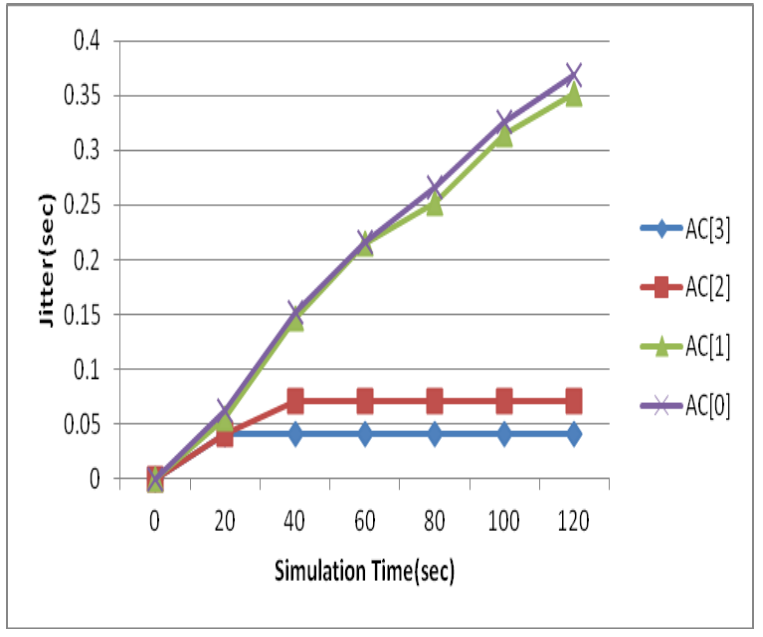

Fig 5: Simulation time Vs Jitter

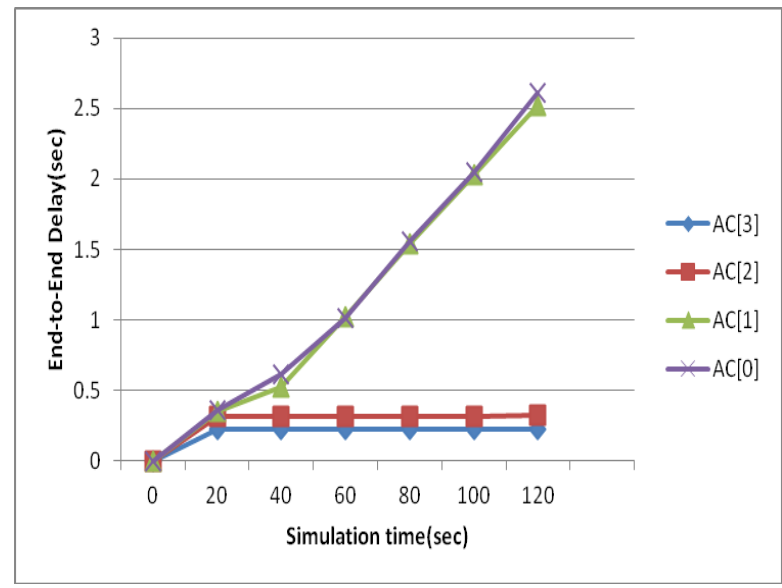

Fig 6: Simulation time Vs E2E delay

By observing Fig.7 whenever no. of transmitting stations are increasing Voice (AC[3]) throughput is increased, remaining AC's throughput decreased.

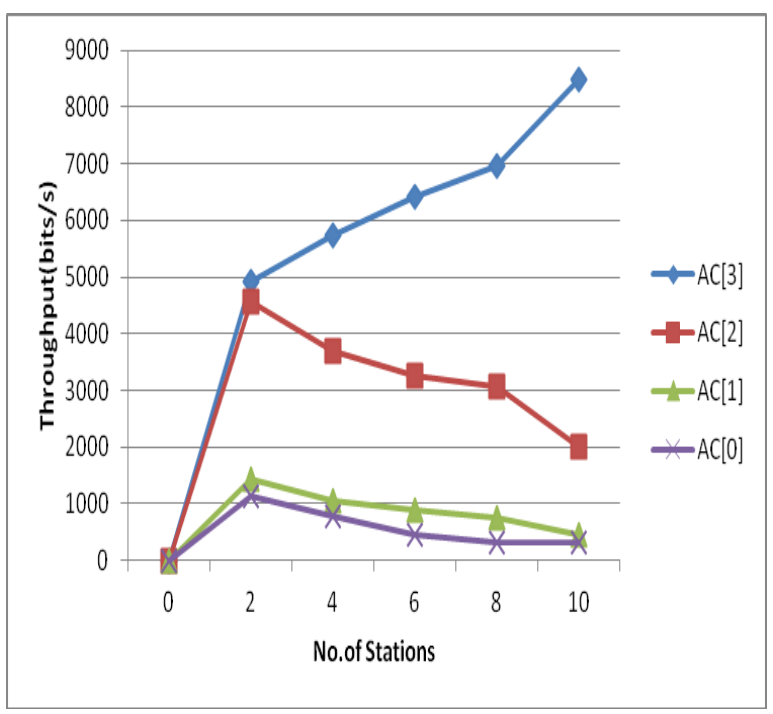

Fig 7: No. of stations Vs Throughput

\section{CONCLUSION AND FUTURE WORK}

This paper shows the Performance of IEEE 802.11 e EDCA in terms of throughput, jitter and E2E delay. By observing the simulation results it is concluded that $\mathrm{AC}[3], \mathrm{AC}[2]$ gets high throughput, low $\mathrm{E} 2 \mathrm{E}$ delay and jitter. $\mathrm{AC}[1], \mathrm{AC}[0]$ gets low throughput, high E2E delay and jitter. Lower-priority traffic is starved by the higher-priority traffic i.e. AC[0], AC[1] are starved by $\mathrm{AC}[2]$ and $\mathrm{AC}[3]$. This is because of static nature of EDCA parameters. By tuning the channel access parameters such as CW size, TXOP limit and AIFS, starvation problem and performance improvement of lower- priority traffic can be solved.

\section{REFERENCES}

[1] ANSI/IEEE. 802.11: Wireless LAN Medium Access Control (MAC) and Physical Layer (PHY) Specifications. IEEE, 1999.

[2] S. Kupa, R.Prakaash "Service Differentiation mechanisms for IEEE 802.11 based wireless networks" IEEE WCNC'04, Pages: 796-801, March-2004. DOI: 10.1.1.58.5318

[3] Wireless medium access control (MAC) and physical layer (PHY) specification: Medium acces control (MAC) enhancements for quality of Service (QoS), IEEE Std. 802.11e/Draft 5.0 (2003).

[4] Tzu Chieh-Tsai, Ming-Ju-Wu "An analytical model for IEEE 802.11e EDCA “ IEEE Conference on Communications, 2005,pages: 3474-3478, ICC-2005. DOI: 10.1109/ICC.2005.1495065

[5] IEEE Draft Standard 802.11e/D13.0 (2005).

[6] Choi, S, Prado, J. D, Shankar, S., Mangold, S. “ IEEE 802.11e contention-based channel access (EDCF) performance evaluation" IEEE ICC'03 ,Vol. 2,Pages: 1151-1156. DOI: 10.1109/ICC.2003.1204546

[7] S. Mangold, S. Choi, G.R. Hierttz, O. Kleein, B.Walke "Analysis of IEEE 802.11e for QoS support in wireless LANs," Proceedings of the IEEE Wireless Communications 2003, vol.10, Pages: 40-50, December2003. DOI: 10.1109/MWC.2003.1265851

[8] http://www.scalable-networks.com

[9] L. Gannoune, S. Robert "Dynamic tuning of the contention window minimum (CWmin) for enhanced service differentiation in IEEE 802.11 wireless ad-hoc networks," Proceedings of the PIMRC 2004, vol. 1, Pages: 311-317, September-2004. DOI: 10.1109/PIMRC.2004.1370885

[10] F.C. Palacio ， J. Majkowski "Dynamic TXOP configuration for Qos enhancement in IEEE 802.11e wireless LAN", International Conference on Software, Telecommunications and Computer Networks SoftCOM-2006.

DOI: 10.1109/SOFTCOM.2006.329721

[11] Ali Hamidian, Ulf Korner "An enhancement to the IEEE 802.11e EDCA providing QoS Guarantees" Telecommun Syst-2006.Volume 31, Issue 2, Pages: 195-212, March2006, springer. DOI: 10.1007/s11235-006-6520-z.

[12] Jelena Mi_si , Saeed Rashwand, Vojislav Mi_si "Analysis of Impact of TXOP Allocation on IEEE 802.11e EDCA under Variable Network Load “ IEEE 
TPDS 2012, vol. 23, no. 5, Pages: 785-799 May-2012. DOI: 10.1109/TPDS.2011.229.

[13] Abdallah Shami, Khalim Amjad Merja "A Collision Avoidance Mechanism for IEEE 802.11e EDCA Protocol to Improve Voice Transmissions in Wireless LANS" IEEE GLOBECOM-2007 proceedings. Pages: 4714-4718, 2007. DOI: 10.1109/GLOCOM.2007.894
[14] Shah Ahsanuzaman Md. Tareq, Fabrozio Grannelli "Performance Analysis of Wireless Ad-Hoc Network Based on EDCA IEEE802.11e "International Journal of Electrical, Computer, Energetic, Electronic and Communication Engineering Vol:4, No:9,Pages: 13221325,2010 